\title{
Self-aligned via and trench for metal contact in III-V semiconductor devices
}

Jun Fei Zheng, Hilmi Volkan Demir, Vijit A. Sabnis, Onur Fidaner, James S. Harris, and David A. B. Miller

Citation: Journal of Vacuum Science \& Technology B: Microelectronics and Nanometer Structures Processing, Measurement, and Phenomena 24, 1117 (2006); doi: 10.1116/1.2188000

View online: http://dx.doi.org/10.1116/1.2188000

View Table of Contents: http://avs.scitation.org/toc/jvn/24/3

Published by the American Institute of Physics 


\title{
Self-aligned via and trench for metal contact in III-V semiconductor devices
}

\author{
Jun Fei Zheng ${ }^{\text {a) }}$ \\ Intel Corporation, Santa Clara, California 95052 \\ Hilmi Volkan Demir \\ Nanotechnology Research Center, Department of Physics, and Department of Electrical and Electronic \\ Engineering, Bilkent University, Bilkent, 06800 Ankara, Turkey
}

Vijit A. Sabnis, Onur Fidaner, James S. Harris, Jr., and David A. B. Miller

E.L. Ginzton Laboratory, Stanford University, Stanford, California 94305

(Received 10 November 2005; accepted 24 February 2006; published 21 April 2006)

\begin{abstract}
A semiconductor processing method for the formation of self-aligned via and trench structures in III-V semiconductor devices (in particular, on InP platform) is presented, together with fabrication results. As a template for such self-aligned via and trench formations in a surrounding polymer layer on a semiconductor device, we make use of a sacrificial layer that consists of either a $\mathrm{SiO}_{2}$ dielectric hard mask layer deposited on the device layers or a sacrificial semiconductor layer grown on top of the device epitaxial layers (e.g., InP on an InGaAs etch stop), both laid down on the device layers before patterning the device geometry. During the semiconductor device etching, the sacrificial layer is kept as a part of the patterned structures and is, therefore, perfectly self-aligned. By selectively removing the sacrificial layer surrounded by the polymer that is etched back within the thickness of the sacrificial layer, an opening such as a via and a trench is formed perfectly self-aligned on the device top area in the place of the sacrificial layer. This process yields a pristine semiconductor surface for metal contacts and fully utilizes the contact area available on the device top, no matter how small the device area is. This approach thus provides as low an Ohmic contact resistance as possible upon filling the via and the trench with metal deposition. The additional use of a thin $\mathrm{Si}_{3} \mathrm{~N}_{4}$ protecting layer surrounding the device sidewalls improves the robustness of the process without any undesired impact on the device electrical passivation (or on the optical mode characteristics if the device also includes a waveguide). This method offers metal contacts scalable to the device size, being limited only by the feasible device size itself. This method is also applicable to the fabrication of other III-V based integrated devices. (C) 2006 American Vacuum Society.
\end{abstract}

[DOI: $10.1116 / 1.2188000]$

\section{INTRODUCTION}

In III-V semiconductor technology, devices are typically fabricated vertically from layered epitaxial materials, where the device top commonly serves as the contact area for metal interconnection. As the devices are scaled down for highspeed operation, it becomes critically important to utilize as large a contact area as possible on the device top for the metal-semiconductor contact so as to achieve a low contact resistance. The traditional approach for making contacts is to etch a via structure through a polymer layer surrounding the III-V devices. ${ }^{1}$ In this case, the via is normally smaller than the device due to the need for a lithography alignment margin. The alignment margin, which is optically and mechanically limited, cannot be further scaled down when the device size is smaller than a certain limit, thus undesirably keeping the via to be a small fraction of the available total device area. Alternative approaches have been used to planarize a polymer film to the device top and use a non-self-aligned metal pad larger than the device for making contact. ${ }^{2,3}$ This approach, however, depends on the ability to planarize the

a) Author to whom correspondence should be addressed; electronic mail: jun.f.zheng@intel.com polymer to level the device top so that the metal does not make contact to the device sidewalls. Not contacting the sidewalls is critical for vertically made layered devices that are sensitive to current leakage and short circuiting on the sidewall. Also, this method would take larger chip area than necessary between devices due to the margin needed for the top non-self-aligned metal pads, in some cases limiting much-needed tight integration between devices, e.g., for short distance interconnects.

In a typical polymer planarization process, for example, with benzocyclobutene (BCB), ${ }^{4}$ the polymer is spun on the entire wafer. Usually this polymer film is so thick that the underlying device height variation will have minimum impact and a planarized polymer top will be achieved. The polymer is then subsequently etched back so that the etched polymer surface is level with that of the devices, allowing the device top to be used for contacts. In so doing, difficulties may arise, especially in wafer-level integrated processing, due to nonuniformities of the spin-on polymer thickness and the etch rate of the polymer across the wafer. Additionally, for example, in the case of monolithic integration, any device height variation due to epitaxial regrowth processes ${ }^{5,6}$ can also lead to the nonplanarization of the polymer on devices across an integrated chip and/or the whole wafer. In 


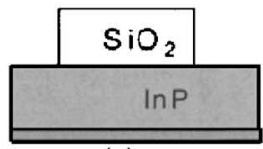

(a)

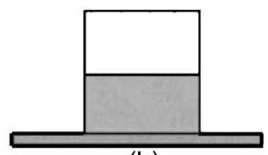

(b)

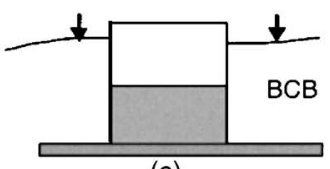

(c)

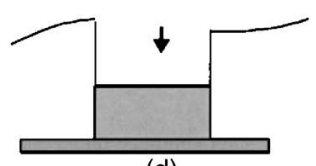

(d)

FIG. 1. Schematics of the self-aligned via (and trench) formation process using a hard mask as a template. (a) The hard mask is formed on the III-V semiconductor. (b) The semiconductor is etched to define the active device area. A proper etch method is used to avoid undercut. (c) Retaining the hard mask, a self-planarizing polymer layer is spun and etched back to stop within the thickness of the hard mask. (d) The hard mask is removed to form a self-aligned via (and trench) opening.

such cases, the exposure of device sidewalls becomes an unavoidable problem in any attempt to clear the polymer from all of the device tops. As a remedy to this problem, a quasiplanarization technique was introduced, which allows for the localized planarization of the device top with the polymer in the close vicinity of the device. ${ }^{7}$ However, this technique relies on an a priori anisotropic semiconductor wet etch process to create an undercut structure beneath the dielectric hard mask used for defining the device mesa. In this technique, it is difficult to control the device dimensions after the anisotropic etch, and such control is especially critical for the devices with dimensions comparable to the undercut created (typically micrometers in size). The micron-scale undercut structure further limits the tight integration of two adjacent devices, thus imposing a severe constraint on how closely they can be integrated on a chip; the undercut margin will keep the devices away from each other, creating a problem similar to that introduced by a non-self-aligned large metal pad, as discussed above.

In this article, we introduce a self-aligned via and trench formation method that allows for the full utilization of the device top for making metal contacts. Providing selfalignment for metallization, we avoid the limitations to tight integration found in previously reported methods. The method eliminates the need for precision lithography for highly accurate alignment of the via. The method yields a pristine surface for making metal-semiconductor contacts. The method is also robust for the wafer-level integration and is insensitive to the aforementioned nonuniformities of spun-on polymer thickness, etch nonuniformity, and device height variation. In Sec. II, we start with presenting the implementation details of the method. In Sec. III, we discuss the application of the method to an InP-based on-chip integration of mesa and waveguide devices for photonic integrated chips, with more advanced and optional variations of the basic method to elaborate the full advantages of the method and its robustness. Finally in Sec. IV, we conclude with a discussion regarding possible future applications.

\section{APPLICATION OF THE METHOD}

The key steps in the process of self-aligned via formation are illustrated in Fig. 1: (a) A hard mask is formed using conventional dielectric thin film deposition, transfer lithography patterning, and etching. This hard mask is required to be thick enough to compensate for the differences in device height, the variation in spin-on polymer thickness, and the nonuniformity in polymer etch back. (b) III-V semiconductor is etched by reactive ion etching (RIE) using the hard mask to define the device. (c) Subsequently, a spin-on polymer layer is spun and etched back to stop within the hard mask for every single device across the wafer. (d) The hard mask is later removed by wet etching, leaving a via structure and a pristine semiconductor surface for metallization. Note that this method can also be applied to generate a trench structure, for example, to be used to metallize the top of a waveguide device such as an electroabsorption modulator (EAM).

In this process, it may be difficult to deposit and pattern thick layers of the dielectric to be used as the hard mask, as dielectric films of micrometers in thickness will introduce an inability to adhere to the wafer and inaccuracy in the lithography pattern transfer process, especially for very small feature sizes down to $100 \mathrm{~nm}$ or below. Moreover, the stress within such a dielectric film can be so high that high-aspectratio hard mask features will peel off mostly because of the relatively higher stress in thick film and the weak adhesion of the dielectric film to small III-V semiconductor structures. To render the process robust in this sense, a sacrificial semiconductor material could be added to the very top in the epitaxial layer design. The sacrificial semiconductor is typi-

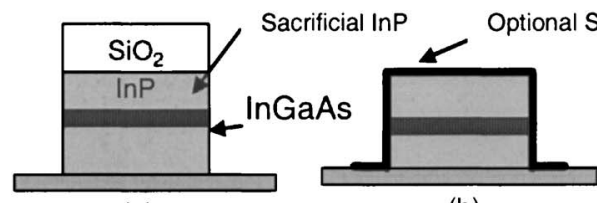

(a) (b)

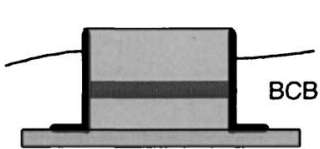

(c)

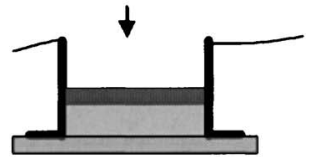

(d)

FIG. 2. Schematics of the self-aligned via (and trench) formation process using sacrificial InP epitaxial material, with InGaAs as an etch-stop layer. (a) The hard mask is used to etch the mesa, including the sacrificial layer. (b) The hard mask is removed. The structure may be protected against wet etch as necessary by covering the wafer surface with a thin $\mathrm{Si}_{3} \mathrm{~N}_{4}$ layer. (c) A thick polymer layer is spun and etched back to stop within the sacrificial layer. (d) The sacrificial layer is selectively wet etched. The etch-stop layer helps render a flat surface, protecting the epitaxial layers beneath. In can be removed using a subsequent selective wet etch, or the etch stop can be retained as the contact layer. 
cally of the same material group as the devices such that it can be epitaxially grown on the device structure and is thus strongly bonded to the device. This will allow for the use of a thinner dielectric mask to pattern the device and, consequently, an accurate pattern transfer is also assured.

This process flow is illustrated in Fig. 2. The sacrificial layer is patterned with the device layers beneath it. The dielectric hard mask is removed using a wet etch. The device structure surface is optionally protected by a layer such as a thin $\mathrm{Si}_{3} \mathrm{~N}_{4}$ layer, which may be necessary to prevent the device structures from being etched away during the subsequent wet etch removal of the sacrificial semiconductor layer. The polymer is then spun in a similar way as described before and the etch back is stopped within the sacrificial semiconductor layer in this case. Then, the sacrificial layer is selectively removed using a wet etch, with the etch-stop layer between the sacrificial layer and the device layers underneath. This forms a via in place of the sacrificial layer. This variation of our method has a number of advantages similar to those of the dielectric hard mask based approach: (i) The sacrificial layer is deposited conveniently during epitaxial growth and can be grown as thick as necessary to accommodate the nonuniformities and to achieve deep enough vias, e.g., as deep as $1 \mu \mathrm{m}$. (ii) The optional sidewall protection is used during the selective wet removal of the sacrificial layer, allowing increased robustness. (iii) Since the sacrificial layer is epitaxially grown, it has good adhesion to the contact material beneath to avoid delaminating from it. (iv) Also, after the removal, it does not leave any dielectric remnant that would increase the contact resistance.

\section{RESULTS AND DISCUSSION}

We employed this method to integrate InP-based waveguide and mesa structures into photonic integrated switches. In the demonstration of a simple process as illustrated in Fig. 1 , a $\mathrm{SiO}_{2}$ hard mask was used along with a $\mathrm{BCB}$ polymer layer $^{2}$ for the via formation. Figure 3 shows the crosssectional scanning electron microscope (SEM) image of one

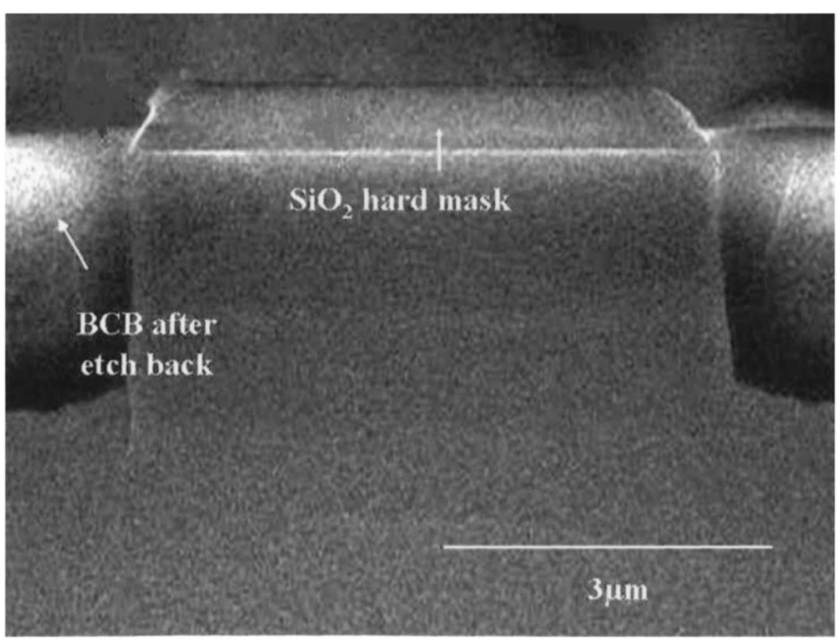

FIG. 3. Cross-sectional SEM image of devices during self-aligned via formation process steps. such device during the process. The thickness of the $\mathrm{SiO}_{2}$ film, which was deposited using a plasma-enhanced chemical-vapor deposition (PECVD) tool, was chosen to be $0.75 \mu \mathrm{m}$ to tolerate wafer-scale nonuniformities. After patterning the $\mathrm{SiO}_{2}$ film to form a hard mask, RIE was used to remove $\mathrm{InP}$ from the unprotected areas to obtain $3 \mu \mathrm{m}$ high waveguide ridges $(4 \mu \mathrm{m}$ wide). Thick BCB polymer layer was spun to yield a $9-10 \mu \mathrm{m}$ thick film after curing to achieve a uniformity of $\pm 0.1 \mu \mathrm{m}$ (excluding an edge of about $2 \mathrm{~mm}$ wide). For the etch back, we used a fast etch recipe with an etch rate of $1.4-1.6 \mu \mathrm{m} / \mathrm{min}$ and a consecutive slow etch recipe with an etch rate of $0.4-0.6 \mu \mathrm{m} / \mathrm{min}$. During the polymer etch back, the thickness of the remaining BCB film could also be monitored using an in situ laser monitor. To ensure that the etch stops within the $\mathrm{SiO}_{2}$ film for all devices across the wafer, the RIE can be ran with additional periodic examinations of the wafer under an optical microscope. We could verify that all of the device tops were cleared by looking for the reappearance of the uniform color of the original $\mathrm{SiO}_{2}$ film at its deposition thickness of $0.75 \mu \mathrm{m}$. Residual $\mathrm{BCB}$ on the $\mathrm{SiO}_{2}$ hard mask will cause a variation of colors due to thin film reflection and interference. Once the device tops are open, the $\mathrm{SiO}_{2}$ hard mask can easily be etched away by an approximately 1 min dip in 6:1 BOE. Consequently, the resulting via is conveniently self-aligned.

From Fig. 3, we notice that the sidewalls of the $\mathrm{SiO}_{2}$ hard mask are sloped. This slope is due to the dielectric etch used to pattern the hard mask. By improving the $\mathrm{SiO}_{2} \mathrm{RIE}$ etch process, the sidewalls can be made straighter. However, this sloped profile can also be advantageous because it makes the $\mathrm{BCB}-\mathrm{SiO}_{2}$ interface tilted, which prevents vertically traveling ions from getting into the $\mathrm{BCB}-\mathrm{SiO}_{2}$ interface. Such undesired penetration of the ions can sometimes result in the so called microtrenching, an etch-enhanced separation of $\mathrm{BCB}$ from the $\mathrm{SiO}_{2}$ or even the InP device beneath it. ${ }^{7}$

Figure 4 shows the atomic force microscope (AFM) top view image of a large $\left(30 \times 30 \mu \mathrm{m}^{2}\right)$ via after the removal of the hard mask. The zoomed image of the surface demonstrates that we can create a pristine surface after the removal of the hard mask. The exposed device surface is a $p+$ contact InGaAs layer, which is atomically flat, except for atomic step

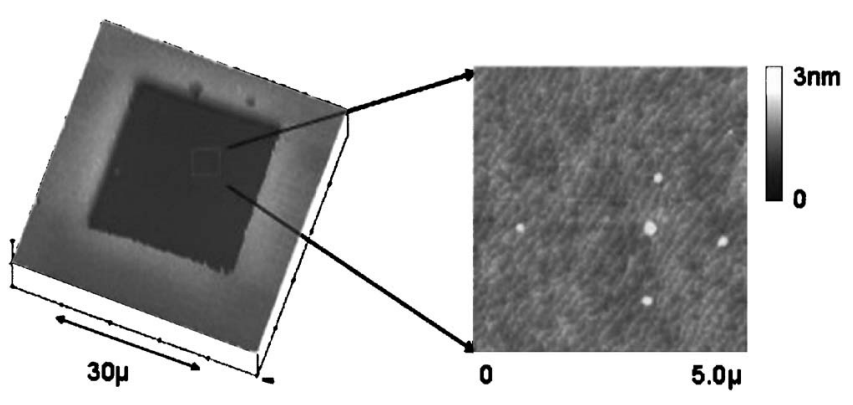

FIG. 4. Atomic force microscope image of a $30 \times 30 \mu \mathrm{m}^{2}$ self-aligned via opening formed after wet etch removal of the $\mathrm{SiO}_{2}$ hard mask. A zoomed image (on the right) of a very small area reveals that a pristine InGaAs surface with clearly visible atomic steps is achieved. 


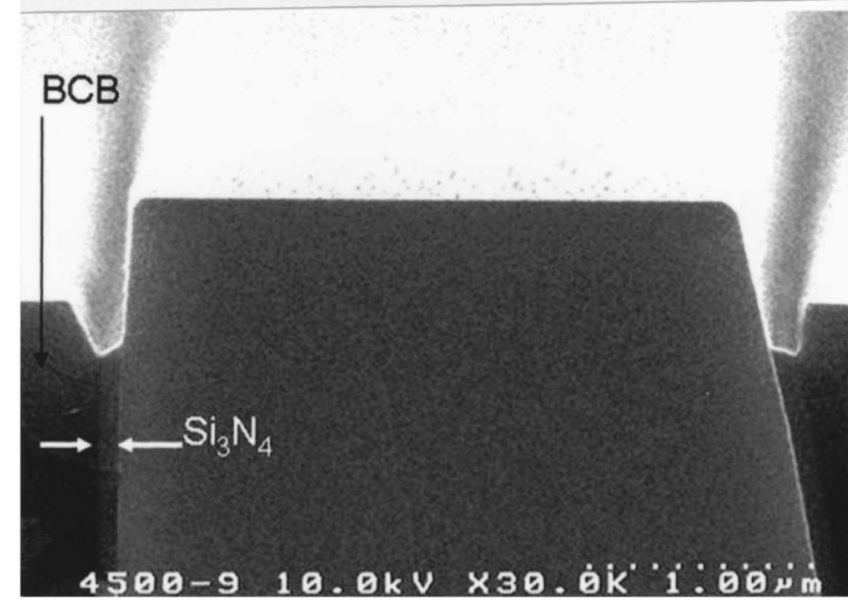

Fig. 5. Cross-sectional SEM picture of InP devices with $\mathrm{Si}_{3} \mathrm{~N}_{4}$ sidewall protection. Note that the top $\mathrm{Si}_{3} \mathrm{~N}_{4}$ layer is etched away during $\mathrm{BCB}$ etch.

features coming from the substrate cut and epitaxial growth. The clear atomic steps in the AFM image indicate that no residue of either $\mathrm{SiO}_{2}$ or $\mathrm{BCB}$ is left on the surface. Note that, in the conventional polymer etch-back method, in which the dielectric hard mask is not retained during the polymer etch, polymer residues usually remain on the top surface due to the difficulty of completely removing the very last polymer on the device surface without overetching the polymer surrounding the device. Also, hydrocarbon molecular deposits may be formed on the device top surface during plasma bombardment or due to heating effect. Such a clean surface obtained using our method allows for very low Ohmic contact resistance. For example, we fabricated diodes and made Ohmic contacts on the diode surfaces prepared by removing $\mathrm{SiO}_{2}$ to achieve contact resistances of $15.3 \pm 3.2 \Omega$ in $30 \times 30 \mu \mathrm{m}^{2}$ device area. ${ }^{7}$

In our second demonstration, we employed an InP sacrificial layer beneath the $\mathrm{SiO}_{2}$ hard mask for the formation of a trench to be used during the metallization of a waveguide structure. In the epitaxial layer design, we used a $50 \mathrm{~nm}$ thick InGaAs layer under the sacrificial InP layer to serve as a wet etch stop during subsequent $\mathrm{InP}$ removal. InGaAs can be allowed to remain in the structure after InP sacrificial layer removal because it can be conveniently used also as the $p+$ contact layer. $\mathrm{A} \mathrm{SiO}_{2}$ hard mask is used, as before, to
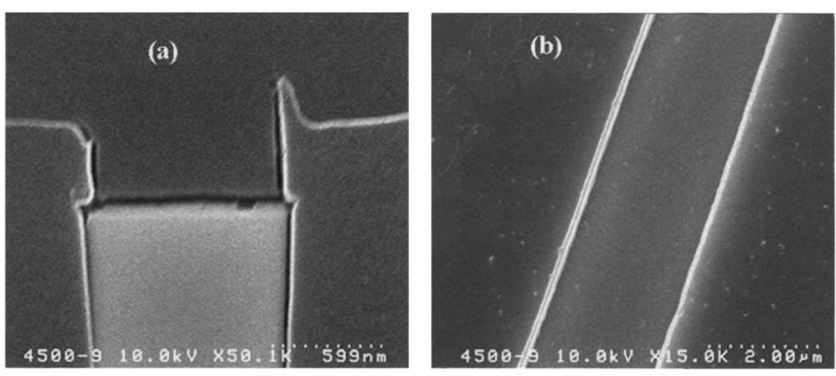

FIG. 6. (a) Cross-sectional SEM picture of a self-aligned trench formed after the wet etch removal of the sacrificial InP and (b) its top view SEM picture along the length of the waveguide.

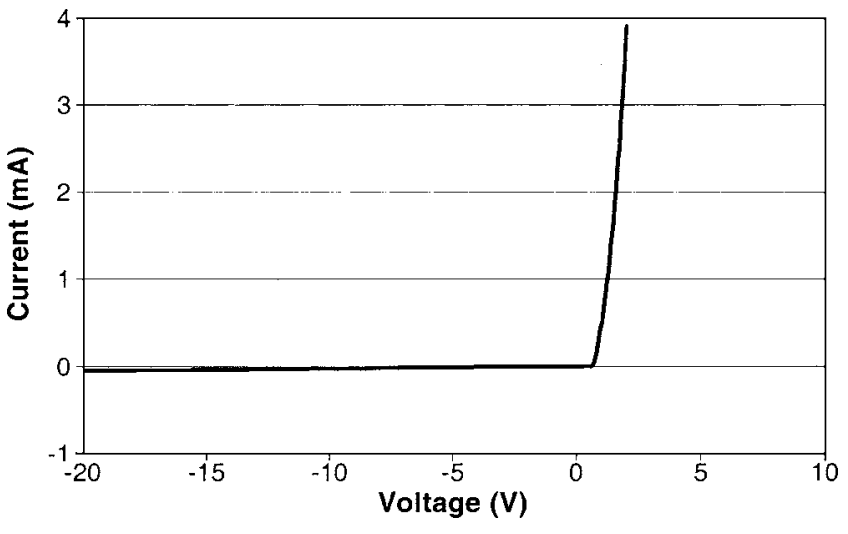

FIG. 7. Measured $I V$ curve of a $\mathrm{Si}_{3} \mathrm{~N}_{4}$ passivated EAM diode. The leakage currents are 8,20 , and $50 \mu \mathrm{A}$ at the reverse biases of 5,10 , and $20 \mathrm{~V}$, respectively, which prove to be low for such a $p-i$ - $n$ diode with a large periphery of $1800 \mu \mathrm{m}^{2}$ sidewall area and an intrinsic layer thinner than $0.5 \mu \mathrm{m}$.

define the waveguide ridge. After defining the waveguide, the $\mathrm{SiO}_{2}$ is removed using the BOE etch. The whole structure after $\mathrm{SiO}_{2}$ removal is, however, protected by the $\mathrm{Si}_{3} \mathrm{~N}_{4}$ thin film deposited before spinning BCB.

This $\mathrm{Si}_{3} \mathrm{~N}_{4}$ thin film has a twofold use: first, it protects any portion of the sidewalls of the devices from exposure to the wet chemical used during the removal of InP sacrificial materials, if the sidewalls are excessively exposed as a result of overetching the polymer $\mathrm{BCB}$ or as a result of having device structures nonuniform in height. The reason for the choice of the $\mathrm{Si}_{3} \mathrm{~N}_{4}$ film stems from its high resistance to strong acidic wet chemical, as in the case of InP removal, for which we used 1:3 $\mathrm{HCl}: \mathrm{H}_{3} \mathrm{PO}_{4}$ mixture. Second, the silicon nitride film protects the device from metal depositing onto device sidewalls in any area where $\mathrm{BCB}$ delaminates from the InP device because of the so called microtrenching. Such microtrenching is due to the enhancement of the $\mathrm{BCB}$ etch at the delaminated interface by the vertically traveling ion bombard during the BCB etch-back process as reported in Ref. 7 and shown in Fig. 5. The $\mathrm{Si}_{3} \mathrm{~N}_{4}$ film adheres very well to the sidewalls of an InP device, so the existing $\mathrm{Si}_{3} \mathrm{~N}_{4}$ strongly protects the sidewall from undesired metal shorting phenomena. The process steps after $\mathrm{Si}_{3} \mathrm{~N}_{4}$ coating are as follows: (1) we spin, cure, and etch back the BCB film; and (2) the $\mathrm{Si}_{3} \mathrm{~N}_{4}$ protection coating on top of the sacrificial $\mathrm{InP}$ is then easily etched away during the $\mathrm{BCB}$ etch back because it is very thin as discussed later, opening the surface of the sacrificial InP for its subsequent etch removal. The InP sacrificial layer after top $\mathrm{Si}_{3} \mathrm{~N}_{4}$ removal is shown in Fig. 5, similar to that previously illustrated in Fig. 2(c). Here Fig. 5 shows a SEM image of the structure during the process steps with a $1000 \AA$ thick $\mathrm{Si}_{3} \mathrm{~N}_{4}$ layer covering the sidewall of the structure before the InP sacrificial layer is removed. Figures 6(a) and 6(b) show the cross-sectional SEM views of the trench over an InP-based waveguide diode after the removal of the sacrificial InP layer. The use of optional $\mathrm{Si}_{3} \mathrm{~N}_{4}$ sidewall protection greatly improves the robustness of the process. 
Contour Map of Transverse Index Proflle

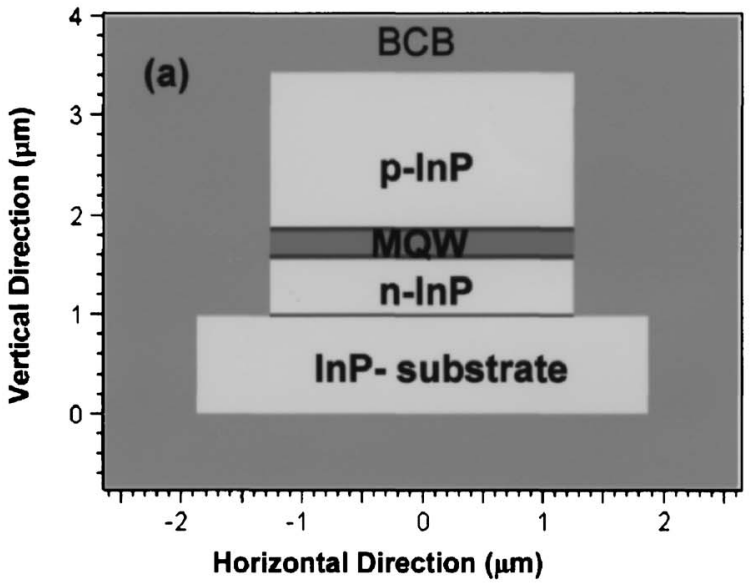

Computed Transverse Mode Profile $\left(m=0, n_{\text {eff }}=3.27276\right)$

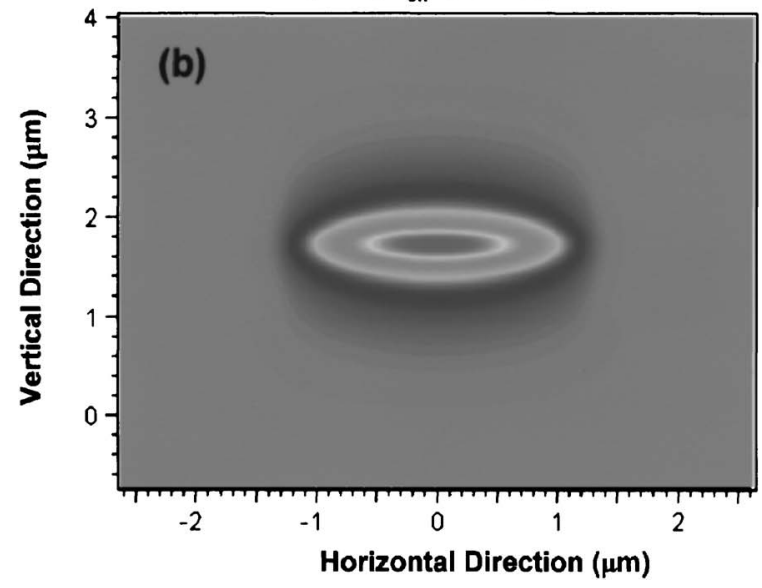

FIG. 8. (a) Cross-sectional illustration of an InP-based waveguide structure without a $\mathrm{Si}_{3} \mathrm{~N}_{4}$ protection surrounded by $\mathrm{BCB}$ and (b) its optical mode at the operating wavelength of $1.55 \mu \mathrm{m}$.

To characterize the effectiveness of $\mathrm{Si}_{3} \mathrm{~N}_{4}$ application, we conducted experiments applying $\mathrm{Si}_{3} \mathrm{~N}_{4}$ with varying thickness ranging from 300 to $1000 \AA$. The thickness of the passivation layer should be chosen in such a way that there is no pin hole formation, hence preventing $\mathrm{HCl}: \mathrm{H}_{3} \mathrm{PO}_{4}(1: 3)$ from attacking the materials under the nitride during the removal of the sacrificial InP layer. We observed that $300 \AA$ of conventional PECVD $\mathrm{Si}_{3} \mathrm{~N}_{4}$ is sufficient for protection during the removal of $1 \mu \mathrm{m}$ thick InP sacrificial material. The $300 \AA \mathrm{Si}_{3} \mathrm{~N}_{4}$ is also sufficient to preserve good passivation of the InP-based device. Figure 7 shows the $I-V$ characteristics of an InP $p-i-n$ waveguide EAM with a $300 \AA$ thick $\mathrm{Si}_{3} \mathrm{~N}_{4}$ sidewall protection. The device has a $0.5 \mu \mathrm{m}$ thick intrinsic layer and it is $300 \mu \mathrm{m}$ long, $3 \mu \mathrm{m}$ deep, and $2 \mu \mathrm{m}$ wide in size.

The film thickness can be increased for even better protection in the case of etching a thicker sacrificial InP layer, provided that such a thick $\mathrm{Si}_{3} \mathrm{~N}_{4}$ film does not have any noticeable undesired impact on the device characteristics, including changing the optical mode in the case of an EAM waveguide. The optical mode can be affected because $\mathrm{Si}_{3} \mathrm{~N}_{4}$
Contour Map of Transverse Index Profile

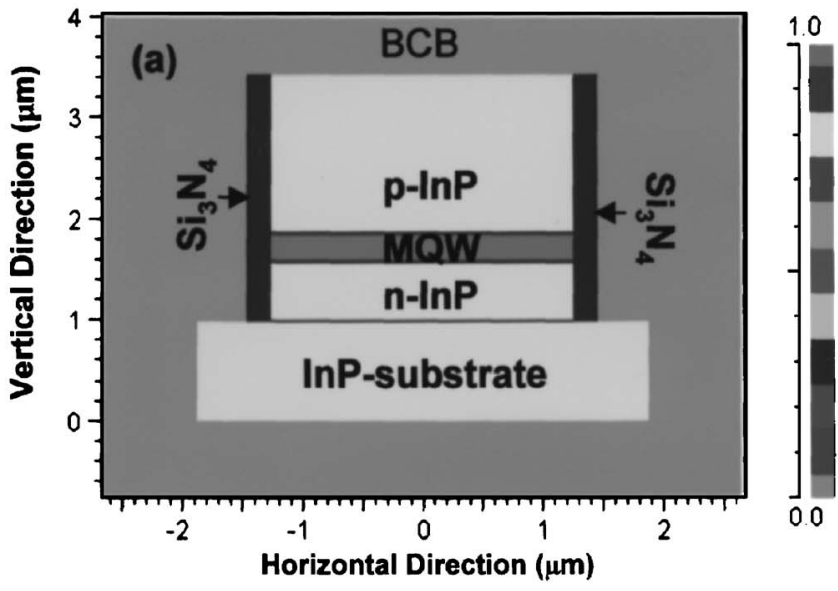

Computed Transverse Mode Profile $\left(m=0, n_{\text {eff }}=3.272923\right)$

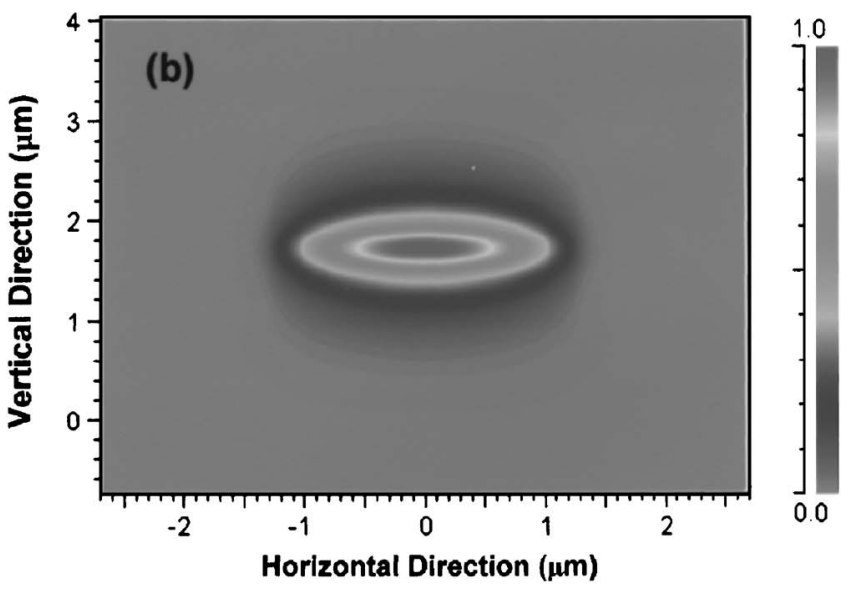

FIG. 9. (a) Cross-sectional illustration of the same InP waveguide structure shown in Fig. 8 except for the addition of a $2000 \AA$ thick $\mathrm{Si}_{3} \mathrm{~N}_{4}$ protection layer surrounded by BCB and (b) its optical mode at $1.55 \mu \mathrm{m}$.

has a different refractive index from that of the surrounding BCB. Our simulation results, as depicted in Figs. 8 and 9, show that even the application of a $2000 \AA$ thick nitride film will not have a substantial impact on the optical mode, which is evidenced by nearly identical modes in Figs. 8(b) and 9(b). We have experimentally demonstrated working waveguide EAM devices with a $1000 \AA$ thick $\mathrm{Si}_{3} \mathrm{~N}_{4}$ sidewall protection. $^{5,6}$

Our method described above, whether employed as it is or in combination with another method, ${ }^{7}$ has proven to be successful in achieving wafer-level integration of InP-based integrated photonic switch devices. ${ }^{5,6}$ Although we have not applied this method to devices implemented in other III-V material systems, such as GaAs, we believe that the method should be applicable to them in principle. Furthermore, if the device sizes call for even smaller via (and trench) formation than the ones presented here, it should still be possible to scale the openings proportionally down, even to nanometer feature sizes in principle, as long as (i) the wet etch removal of nanometer size oxide hard mask (or InP sacrificial layer, 
for that matter) is still possible and (ii) the $\mathrm{Si}_{3} \mathrm{~N}_{4}$ protection layer does not induce any adverse effect due to its minimum thickness requirements. The key aspect of the method presented here is that it is self-aligning and so the scalability of via (and trench) formation is only limited to the capability of fabricating the desired device size. Using the method described above, we have successfully fabricated and demonstrated optical devices integrated with waveguide EAMs that have widths of only $1-2 \mu \mathrm{m} .^{8,9}$

\section{CONCLUSIONS}

We presented a processing technique for self-aligned via and trench formations that can use the full area of the device tops for metallization and, thus, maximizes the area for electrical contact and minimizes contact resistance. Because this method is self-aligned to semiconductor structures using either a hard mask or a sacrificial semiconductor layer in the structure as a template, it is in principle scalable to much smaller dimensions as long as devices of smaller sizes can be made. We demonstrate that the method is successfully applied to InP-based $30 \times 30 \mu \mathrm{m}^{2}$ mesa and $2 \times 300 \mu \mathrm{m}^{2}$ waveguide devices. We show that the method yields a pristine semiconductor surface for metallization. The robustness of the process is substantially improved and the device is well passivated by using an optical $\mathrm{Si}_{3} \mathrm{~N}_{4}$ sidewall protection layer, without a noticeable change induced in the optical characteristics of the waveguide devices.

\section{ACKNOWLEDGMENTS}

This research is supported by Intel Corporation. The authors acknowledge Dr. Jesper Hanberg for the epitaxial growth of the InP samples.

${ }^{1}$ C. P. Wong, Polymers for Electronic and Photonic Applications (Academic, San Diego, CA, 1993).

${ }^{2}$ Y.-H. So, P. Garrou, J.-H. Im, and D. M. Scheck, Chem. Innovation 31 , 40 (2001).

${ }^{3}$ J. D. Robinson and B. Humphreys, "Plasma etch optimization of a photo sensitive benzocyclobutene (BCB) planarization layer for photonic integrated circuit manufacturing," MetroPhotonics, Inc. Technical Report, April 2003.

4"Processing procedures for Dry-Etch Cyclotene Advanced Electronics Resin," Dow Chemicals Technical Report.

${ }^{5}$ H. V. Demir, V. A. Sabnis, O. Fidaner, J. S. Harris Jr., D. A. B. Miller, and J. F. Zheng, IEEE J. Sel. Top. Quantum Electron. 11, 86 (2005).

${ }^{6}$ V. A. Sabnis, H. V. Demir, O. Fidaner, J. S. Harris Jr., D. A. B. Miller, J. F. Zheng, N. Li, T.-C. Wu, H.-T. Chen, and Y.-M. Houng, Appl. Phys. Lett. 84, 469 (2004).

${ }^{7}$ H. V. Demir, J. F. Zheng, V. A. Sabnis, O. Fidaner, J. P. Hanberg, J. S. Harris Jr., and D. A. B. Miller, IEEE Trans. Semicond. Manuf. 18, 182 (2005).

${ }^{8}$ O. Fidaner, H. V. Demir, V. A. Sabnis, J. S. Harris, D. A. B. Miller, and J. F. Zheng, IEEE Lasers and Electro-optics Society Annual Meeting, 7-11 November 2004, Rio Grande, Puerto Rico (unpublished), Paper WA1.

${ }^{9}$ O. Fidaner, H. V. Demir, V. A. Sabnis, J. F. Zheng, J. S. Harris, Jr., and D. A. B. Miller, Opt. Express 14, 361 (2006). 